УДК 517.53

\title{
An Approach to Determine the Resultant of Two Entire Functions
}

\author{
Olga V. Khodos* \\ Institute of Mathematics and Computer Science \\ Siberian Federal University \\ Svobodny, 79, Krasnoyarsk, 660041 \\ Russia
}

Received 06.12.2017, received in revised form 19.01.2018, accepted 06.03.2018

An approach to determine the resultant of two entire functions is studied.

Keywords: resultant, entire function of finite order of growth.

DOI: 10.17516/1997-1397-2018-11-2-264-268.

Classical recurrent Newton's identities give relations between sums of powers of the roots of a polynomial and the coefficients of this polynomial (see, e.g., [1-3]). These formulas can be obtained with the use of the Cauchy integral formula [4, Ch.1]. This fact allows us to expand the class of functions for which these recurrent formulas are valid. Namely, for the class of entire functions of finite order of growth one can obtain relations between the coefficients of a Taylor expansion of a given function and sums of negative powers of zeros of the function [4, Ch.1].

Using the methods of complex analysis, we introduce the concept of the resultant for an entire function and an entire function with finite number of zeros and establish its properties. The proposed approach can be useful, for example, in studies of equations of chemical kinetics where exponential polynomials arise [5,6]. It also allows us to apply this approach to the elimination of unknowns from systems of non-algebraic equations on the basis of the Zel'dovich-Semenov scheme [7].

Let us consider two polynomials $f$ and $g$. The classical resultant $R(f, g)$ can be defined in several ways:

a) by using the Sylvester determinant (see, e.g., [1-3]);

b) by virtue of the product formula $R(f, g)=\prod_{\{x: f(x)=0\}} g(x)$ (see, e.g., [1-3]);

c) by using the Bézout-Cayley formula (see, e.g., [8]).

The product formula and the Sylvester resultant are used in the proposed approach. It is motivated by the fact that entire functions are natural generalization of polynomials in complex analysis.

A number of papers [9-13] has been devoted to the generalization of the notion of resultant for analytic functions in annuli, matrices, meromorphic functions on a complex Riemann surface and for systems of algebraic equations. However, it is assumed in all these studies that the number of zeros or singular points (poles) is finite. We assume that an entire function can have an infinite number of zeros. The downside of this is that a closed formula can not be obtained.

An approach to determine the resultants of two entire functions was proposed [14]. The case when one of the functions is entire, and the second is a polynomial (or an entire function with a finite number of zeros) was considered.

Let $f(z)$ and $g(z)$ be entire functions on complex plane $\mathbb{C}$ of the form

$$
f(z)=a_{0}+a_{1} z+a_{2} z^{2}+\ldots+a_{n} z^{n}+\ldots, \quad a_{0}=1,
$$

*khodos_olga@mail.ru

(c) Siberian Federal University. All rights reserved 
and

$$
g(z)=b_{0}+b_{1} z+b_{2} z^{2}+\ldots+b_{n} z^{n}+\ldots .
$$

In what follows we consider transcendental functions $f(z)$ and $g(z)$, that is, entire functions for which infinitely many coefficients $a_{j}$ and $b_{j}$ are not equal to zero. It is of interest to find out when these functions have at least one common zero.

If $f$ and $g$ are polynomials then the answer is given by the Sylvester classical resultant (see, for example, [2]). The case when $f(z)$ is a polynomial, and $g(z)$ is a transcendental function was considered before [14]. The purpose of this paper is to consider transcendental functions $f$ and $g$.

The main approach to solve this problem is based on the formula for the logarithmic residue (see, for example, [15]).

Let $\gamma_{R}=\{z:|z|=R\}$ be a circle centred at the origin and of radius $R$. Suppose that zeros of $f(z)$ do not lie on $\gamma_{R}$. Let us consider the following integral

$$
I_{R}=\frac{1}{2 \pi i} \int_{\gamma_{R}} g(\zeta) \frac{d f(\zeta)}{f(\zeta)} .
$$

By the formula of the logarithmic residue (see, for example, [15]), the integral is equal to

$$
I_{R}=\sum_{k=1}^{N_{R}} g\left(\alpha_{k}\right),
$$

where $N_{R}$ is the number of zeros of $f(z)$ in the disk $C_{R}=\{z:|z|<R\}$ (zeros are counted together with their multiplicities), and $\alpha_{k}$ are zeros.

If we consider integrals

$$
I_{R}^{m}=\frac{1}{2 \pi i} \int_{\gamma_{R}} g^{m}(\zeta) \frac{d f(\zeta)}{f(\zeta)}, \quad m \in \mathbb{N}
$$

then

$$
I_{R}^{m}=\sum_{k=1}^{N_{R}} g^{m}\left(\alpha_{k}\right) .
$$

Applying the classical Newton recurrence formulas (see, for example, [2]) to the sequence $I_{R}^{m}$, $m=1, \ldots, N_{R}$, one can obtain the following expression

$$
\prod_{s=1}^{N_{R}} g\left(\alpha_{s}\right) .
$$

Thus, if expression (4) is equal to zero then functions $f(z)$ and $g(z)$ have common zeros in the disk $C_{R}$.

If we now increase $R$ unlimitedly then two options are possible: either for some $R$ expression (4) tends to zero, i.e., $f$ and $g(z)$ have a common zero in $C_{R}$; or expression (4) does not tend to zero for any $R$, i.e., $f$ and $g(z)$ do not have common zeros in $\mathbb{C}$.

Thus, to construct the resultant of functions $f(z)$ and $g(z)$ one need to compute integrals (3) and (4) for any $R$ without finding zeros of function $g(z)$. Here we consider one approach to solve this problem.

Let $P_{n}(z)$ be the Taylor polynomial of $f(z)$ of order $n$, that is,

$$
P_{n}(z)=a_{0}+a_{1} z+a_{2} z^{2}+\ldots+a_{n} z^{n},
$$

and $Q_{n}(z)$ is the remainder $f(z)-P_{n}(z)$. 
Let us suppose that for a given $n$ there exists $R>0$ such that

$$
\left|P_{n}(z)\right|>\left|Q_{n}(z)\right|, \quad \text { если }|z|=R,
$$

i.e., inequality (5) holds on the circle $\gamma_{R}=\{z:|z|=R\}$, and in addition all the roots of polynomial $P_{n}(z)$ lie in the disk $C_{R}$. In this case integrals of the form (3) can be calculated in terms of the Taylor coefficients of functions $f(z)$ and $g(z)$.

Since for sufficiently large $R$ the following inequality holds (lemma on the modulus of the highest term)

$$
\left|P_{n-1}(z)\right|<\left|a_{n}\right| R^{n} .
$$

If $a_{n} \neq 0$ then by the Rouché theorem polynomial $P_{n}(z)$ has exactly $n$ roots in the disc $C_{R}=\{z:|z|<R\}$ (counted together with their multiplicities), and it has no roots outside this circle

Therefore, in what follows we assume that for a given $R$ conditions (5) and (6) hold. Then all roots of polynomial $P_{n}(z)$ lie in the disk $C_{R}$.

Let us consider the following integral

$$
J_{s}=\frac{1}{2 \pi i} \int_{\gamma_{R}} \zeta^{s} \frac{d \zeta}{P_{n}(\zeta)}, \quad s \in \mathbb{N} .
$$

Using the change of variables $\zeta \rightarrow 1 / w$ in integral (7), we obtain

$$
J_{s}=-\int_{\gamma_{\frac{1}{R}}} \frac{1}{w^{s}} \cdot \frac{d\left(\frac{1}{w}\right)}{P_{n}\left(\frac{1}{w}\right)} .
$$

Since $P_{n}(\zeta)=a_{0}+a_{1} \zeta+\ldots+a_{n} \zeta^{n}$ then

$$
P_{n}(1 / w)=a_{0}+a_{1} / w+\ldots+a_{n} / w^{n}=\frac{1}{w^{n}}\left(a_{0} w^{n}+a_{1} w^{n-1}+\ldots+a_{n}\right)=\frac{1}{w^{n}} P_{n}^{*}(w)
$$

where $P_{n}^{*}(w)=a_{0} w^{n}+a_{1} w^{n-1}+\ldots+a_{n}$. Taking into account condition (5), polynomial $P^{*}(w)$ has no roots in the disk $C_{\frac{1}{R}}$. We have

$$
J_{s}=\int_{\gamma_{\frac{1}{R}}} \frac{1}{w^{s}} \cdot \frac{d w}{w^{2}} \cdot \frac{1}{\frac{1}{w^{n}} \cdot P_{n}^{*}(w)}=\int_{\gamma_{\frac{1}{R}}} w^{n-s-2} \cdot \frac{d w}{P_{n}^{*}(w)} .
$$

Hence we obtain the following assertion.

Theorem 1. If $n-s-2 \geqslant 0$ then $J_{s}=0$. If $n-s-2<0$ then

$$
J_{s}=\left.\frac{2 \pi i}{(s-n) !} \cdot \frac{\partial^{s+1-n}}{\partial w^{s+1-n}} \frac{1}{P_{n}^{*}(w)}\right|_{w=0} .
$$

So with condition (5), integral $J_{s}$ can be calculated in a finite form. This theorem is the basis for finding the resultant of two entire functions.

Let us now consider the fulfilment of conditions (5) and (6).

We give an estimate of $R$ for which inequality (6) holds. Obviously, inequality (6) holds if the inequality

$$
\sum_{k=0}^{n-1}\left|a_{k}\right| R^{k}<\left|a_{n}\right| R^{n}
$$


holds.

Assuming $R>1$, we obtain that inequality (6) holds if the inequality

$$
\sum_{k=0}^{n-1}\left|a_{k}\right|<\left|a_{n}\right| R
$$

holds. Thus the following assertion is valid.

Theorem 2. Let $a_{n} \neq 0$, and the inequality

$$
R>\frac{\sum_{k=0}^{n-1}\left|a_{k}\right|}{\left|a_{n}\right|},
$$

holds for $R>1$. Then all roots of polynomial $P_{n}(z)$ lie in the disk $C_{R}$.

Since

$$
\sum_{k=0}^{n-1}\left|a_{k}\right| \leqslant \sum_{k=0}^{\infty}\left|a_{k}\right|=K
$$

then the following is valid.

Corollary 1. Let $a_{n} \neq 0$. If for some $R$ the inequality

$$
R>\frac{K}{\left|a_{n}\right|}
$$

holds then all roots of $P_{n}(z)$ lie in the disk $C_{R}$.

Let us find conditions on $R$ when the inequality $\left|P_{n}(z)\right|>\varepsilon\left|a_{n}\right| R^{n}$ holds for some $0<\varepsilon<1$, i.e.,

$$
\left|a_{0}+a_{1} z+\ldots+a_{n} z^{n}\right|>\varepsilon\left|a_{n}\right| R^{n} .
$$

It follows from (6) that

$$
\left|P_{n}(z)\right| \geqslant\left|a_{n}\right| R^{n}-\left|P_{n-1}(z)\right|>0 .
$$

Then for $0<\varepsilon<1$ and for some sufficiently large $R$ the inequality

$$
\left|a_{n}\right| R^{n}-\left|P_{n-1}(z)\right|>\varepsilon\left|a_{n}\right| R^{n}
$$

holds, i.e.,

$$
\left|a_{n}\right|(1-\varepsilon) R^{n}>\left|P_{n-1}\right| .
$$

Theorem 3. If for some $R$ the inequality

$$
R>\frac{\sum_{k=0}^{n-1}\left|a_{k}\right|}{\left|a_{n}\right|(1-\varepsilon)}
$$

holds then conditions (6) and (10) are satisfied.

Corollary 2. If

$$
R>\frac{K}{\left|a_{n}\right|(1-\varepsilon)}
$$

then conditions (6) and (10) are satisfied.

This research was supported by the Russian Foundation for Basic Research (grant 15-0100277) and by the grant of the Government of the Russian Federation for research under the guidance of leading scientist of Siberian Federal University (contract 14.Y26.31.0006). 


\section{References}

[1] B.L.Van der Waerden, Algebra, v. 1, New York, Springer, 1950.

[2] A.G.Kurosh, Course in higher algebra, Moscow, Nauka, 1968 (in Russian).

[3] N.Bourbaki, Algébre. Livre 2, Paris, Hermann, 1961.

[4] V.I.Bykov, A.M.Kytmanov, M.Z. Lazman, Elimination Methods in Polynomial Computer Algebra, Basel, Kluwer Academic Publishers, 1998.

[5] V.I.Bykov, Modeling of the critical phenomena in chemical kinetics, Moscow, Komkniga, 2006 (in Russian).

[6] V.I.Bykov, Tsybenova S.B. Non-linear models of chemical kinetics, Moscow, KRASAND, 2011 (in Russian).

[7] O.V.Khodos, On zeros of holomorphic functions, Journal of Siberian Federal University. Mathematics \& Physics, 9(2016), no. 3, 307-309.

[8] M.G.Krein, M.A.Naimark, The method of symmetric and Hermitian forms in the theory of the roots of algebraic equation, Linear and Multilinear Algebra, 10(1981), 265-308.

[9] I.C.Gohberg, G.Heinig, Resultant matrix and its generalization. I. Resultant operator of matrix polynomal, Acta Sci. Math., 37(1975), 41-61.

[10] I.C.Gohberg, G.Heinig, Resultant matrix and its generalization. II. Continual analog of resultant matrix, Acta Math. Acad. Sci. Hungar., 28(1976), 189-209.

[11] I.C.Gohberg, L.E.Lere,r Resultant operators of a pair of analytic functions, Proc. Math. Amer. Soc., 72(1978), no. 1, 65-73.

[12] B.Gustafsson, V.G.Tkachev, The resultant on compact Riemann surfaces, Comm. Math. Physics, 10(2009), 265-308.

[13] A.Yu.Morozov, Sh.R.Shakirov, New and old resultant in resultant theory Theor. math. Physics, 163(2010), no. 2, 587-617.

[14] A.M.Kytmanov, Ya.M.Naprienko, One approach to finding the resultant of two entire functions, Complex variables and elliptic equations, 62(2017), no. 2, 269-286.

[15] B.V.Shabat, Introduction to complexe analysis, v. I, AMS, Providence, 1990.

\section{Подход к определению результанта двух целых функций}

Ольга В. Ходос

Институт математики и фундаментальной информатики

Сибирский федеральный университет Свободный, 79, Красноярск, 660041

Россия

Исследуется подход к определению результанта двух цельх функций.

Ключевые слова: результант, целая функиия конечного порядка роста. 